J. Lake Sci. (湖泊科学), $2007, \mathbf{1 9 ( 3 ) ~ : 2 4 1 - 2 4 9 ~}$

http:// www. jlakes. org. E-mail: jlakes@ niglas. ac.cn

(c) 2007 by Journal of Lake Sciences

\title{
分季节的太湖悬浮物遥感估测模型研究
}

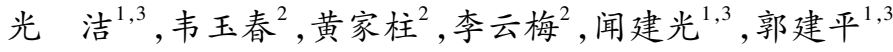 \\ (1: 中国科学院遥感应用研究所遥感科学国家重点实验室, 北京 100101) \\ (2: 南京师范大学地理科学学院,南京 210097) \\ (3: 中国科学院研究生院, 北京 100039)
}

摘 要: 根据 $1996-2002$ 年无锡太湖监测站的水质资料分析,太湖悬浮物具有季节性特征,因而分季节的悬浮物估测模 型比单一的模型可能更加适合用来估测太湖全年的悬浮物浓度. 在分析太湖水体光谱特征的基础上,根据太湖悬浮物的 季节性分布特征,使用春夏秋冬四季的 Landsat TM/ETM 图像和准同步的水质采样数据,建立了太湖分季节的悬浮物估算 模型. 结果表明: 估测因子 $(B 2+B 3) /(B 2 / B 3)$ 在春、秋、冬三季都能很好地估测出悬浮物的浓度 $\left(R^{2}>0.52\right)$. 夏季由于 叶绿素的干扰性较大, 悬浮物的估测效果不理想. 冬季的估测效果最好 $\left(R^{2}=0.81\right)$, 模型为 $\ln S S=14.656 \times(B 2+B 3) /$ $(B 2 / B 3)+1.661$, 其中, $\ln S S$ 表示悬浮物取自然对数后的值, $B 2 、 B 3$ 为 TM/ETM 图像经过 $6 \mathrm{~S}$ 大气校正、 $3 \times 3$ 低通滤波后 第 $2 、 3$ 波段的反射率值.

关键词:悬浮物; 分季节模型; 遥感;实测光谱;太湖

\section{Seasonal suspended sediment estimating models in Lake Taihu using remote sensing data}

GUANG Jie $^{1,3}$, WEI Yuchun ${ }^{2}$, HUANG Jiazhu ${ }^{2}$,LI Yunmei ${ }^{2}$, WEN Jianguang ${ }^{1,3} \&$ GUO Jiangping ${ }^{1,3}$

( 1 : State Key Laboratory of Remote Sensing Science, Jointly Sponsored by the Institute of Remote Sensing Applications of Chinese Academy of Sciences and Beijing Normal University, Institute of Remote Sensing Applications, CAS, Beijing100101,P. R. China)

(2: College of Geographical Science, Nanjing Normal University, Nanjing 210097,P. R. China)

(3: Graduate School of the Chinese Academy of Sciences, Beijing 100039, P. R. China)

Abstract: Suspended sediment in Lake Taihu has its seasonal character according to the analysis of in situ data acquired by Taihu Monitoring Wuxi Station during 1996-2002, so seasonal models may better than a single model for estimating suspended sediment in Lake Taihu. After analyzing the spectral characteristic of Lake Taihu, seasonal suspended sediment estimating models were built based on four Landsat TM/ETM images, respectively in spring, summer, autumn and winter, as well as synchronous in situ data. Result shows that $(B 2+B 3) /(B 2 / B 3)$ is a good index for estimating suspended sediment in Spring, Autumn and Winter $\left(R^{2}>0.52\right)$. The summer model is not sound due to the disturbance of high chlorophyll concentration, as alga boom in summer. The winter model has the best effect in estimating suspended sediment $\left(R^{2}=0.81\right)$. The Winter model is $\ln S S=14.656 \times(B 2+B 3) /(B 2 / B 3)+$ 1. 661 , in which $\ln S S$ is the natural logarithm of suspended sediment concentration, $B 2$ and $B 3$ are the reflectance in Band 2 and B3 of the Landsat TM/ETM images after $6 \mathrm{~S}$ atmospheric correction and a $3 \times 3$ low-pass filtering.

Keywords : suspended sediment; seasonal model; remote sensing; field spectral; Lake Taihu

在水体中悬浮物的存在是相当普遍的现象, 悬浮物含量的多少直接影响到光在水体中的传播, 进而影 响着水生生态环境, 最终决定了湖泊的初级生产力 ${ }^{[1]}$. 悬浮物的时空分布状况和运动规律的研究还直接关 系到正确估算水土流失、航道港口的冲淤变化、近岸水产养殖开发等重要问题 ${ }^{[2]}$. 常规的悬浮物监测方法

* 国家“863”计划项目 (2003AA131060) 和国家自然科学基金 (40571110,40471091) 联合资助. $2006-08$-06 收稿; $2006-11-01$ 收修改稿. 光洁, 女, 1982 年生, 博士研究生;E-mail : guangjier@163.com. 
受人力、物力和气候、水文条件的限制, 难以长时间跟踪监测, 且悬浮物的空间分布信息难以从日常的环境 监测中获取. 遥感监测则可以大面积、迅速地提供水质信息, 以其特有的优势成为持久监测区域乃至全球 尺度上湖泊、水库的有效手段.

二类水体水质遥感监测利用经验、半经验或分析的方法,选择合适的遥感波段数据,建立水质参数遥感 估测模型估算水体中水质参数浓度 ${ }^{[3]}$. 半经验方法是自 20 世纪 90 年代以来最常用的水质参数遥感反演 方法. 国内外很多学者 ${ }^{[4-15]}$ 多利用这种方法监测湖泊、水库的水质参数如总悬浮物、叶绿素及与之相关的 透明度、浑浊度和富营养化指数等, 研究的湖泊遍及欧洲、北美、澳洲. 该方法由于对特定水体进行了实地 光谱测量以确定最佳反演波段或波段组合,再建立水质参数与光谱或光谱变化式 (如对数、一阶微分等) 的 回归方程,因而具有一定的物理意义. 然而,由于二类水体中信息复合因素多,专题信息提取的难度高, 以 及悬浮物颗粒大小、组成等的不同导致悬浮物的遥感估测模型在模型形式和波段选择上随着水域环境的变 化有所差异 ${ }^{[7]}$. Williams ${ }^{[8]}$ 于 1973 年就对切萨比克湾进行了悬浮物的遥感定量工作, 发现了悬浮物与卫星 遥感数据呈线性关系. 1974 年 Klemas 等人 ${ }^{[9]}$ 将遥感资料应用于特拉华湾, 发现悬浮物含量与陆地卫星 MSS 亮度值呈对数关系. 1979 年 Munday 和 Alfoldi ${ }^{[10]}$ 研究了芬地湾, 认为对数反演模式要比线性反演模式 好. Chen ZM 等 ${ }^{[11]}$ 对 18 种不同浓度不同类型不同粒径的悬浮物在 $350-2500 \mathrm{~nm}$ 范围的光谱特征研究结 果表明在 $450-700 \mathrm{~nm}$ 波段范围,悬浮物浓度与反射率是一种对数线性关系, 而在 $700-1015 \mathrm{~nm}$ 波段范围 呈线性关系. Lathrop ${ }^{[12]}$ 使用 $B 2 / B 1$ 和 $B 3 / B 1$ 来反演内陆混浊水体的悬浮物浓度, Populus 等 ${ }^{[13]}$ 在印度尼 西亚海区使用 $B 3 / B 2$ 作为反演悬浮物的变量. 在针对太湖水体悬浮物的研究中, 由于研究时间、采样点布 设等的不同,各研究者的研究结果不尽相同. 如王学军等 ${ }^{[14]}$ 提出使用 TM 图像的 $(B 3+B 4) /(B 1+B 2)$ 作 为反演悬浮物的变量; 吕恒 ${ }^{[15]}$ 使用了 $B 3-B 1$ 估测太湖悬浮物浓度;马荣华等 ${ }^{[6]}$ 的研究则表明用 $B 4 / B 1$ 估 算悬浮物含量的精度最高; 刘堂友等 ${ }^{[16]}$ 通过机载成像光谱仪、水面高光谱测量数据及水样测试结果的综合 分析,认为估测悬浮物的最佳波段组合为红波段和蓝波段; Wei YC 等 ${ }^{[17]}$ 提出了悬浮物的归一化指数模型; Zhou $\mathrm{W}$ 等 ${ }^{[18]}$ 使用了两幅 $\mathrm{TM}$ 图像, 认为 $B 3$ 最适合于不同水质情况下的悬浮物估测 $(B 1 、 B 2 、 B 3 、 B 4$ 分别为 TM/ETM 图像 1-4 波段的反射率). 与海洋及其他深水湖泊相比,太湖中悬浮物浓度较高, 全湖多年平均 值为 $34.32 \mathrm{mg} / \mathrm{L}$, 湖内的不同湖区, 悬浮物浓度差异很大. 由于不同季节, 风速、风向不一样, 加之动植物季 节生长差异, 不同季节湖水中的悬浮物组成 (无机、有机颗粒物比例) 也存在差别 ${ }^{[19]}$. 由于悬浮物存在着季 节性差异, 建立分季节的悬浮物估测模型比单一的模型可能更加适合用来估测太湖全年的悬浮物浓度. 本 文根据太湖悬浮物的季节性分布特征,利用分布于四个季节的 Landsat TM/ETM 图像,在充分考虑悬浮物光 谱特征的基础上, 建立分季节的悬浮物遥感估测模型, 以适应不同的季节条件, 提高太湖水体悬浮物浓度的 估算精度.

\section{1 数据获取与预处理}

\section{1 水质分析数据及样点分布}

地面水质资料有无锡太湖监测站 1996-2002 年各月的常规站点的水质资料 (共 19 个样点), 根据遥感 影像的同步性要求,选用了与遥感影像成像日期最接近的 2000 年 5 月 $8-14$ 日、2001 年 1 月 $8-15$ 日、 2002 年 9 月 2-6日的测量数据;2003 年 11 月 23 日中国科学院南京地理与湖泊研究所的实测数据(共 21 个样点), 样点的位置和编号见图 1. 悬浮物浓度的测量均采用烘干称重法 (GB11901-89 标准) 测定, 单位 为 $\mathrm{mg} / \mathrm{L}$.

\section{2 实测光谱数据}

2003 年 11 月 13 日在太湖采集了 16 个点的水面反射光谱数据,用 GPS 获取采样点位置信息 (图 1 ). 光谱测量采用美国 ASD 公司生产的 FieldSpec HandHeld 手持式地物光谱仪,其波长范围为 $325-1075 \mathrm{~nm}$, 共 512 个波段. 采样当天, 天空晴朗无云, 湖面平静. 测量水体之前先用光谱仪测一下标准参考板, 然后距 离水面 $1 \mathrm{~m}$ 处垂直水面进行测量, 每个采样点至少测量 10 次光谱, 剔除由于毛细波的太阳直射反射造成的 数值较高的曲线, 保留数值较低的曲线进行平均, 作为该点的光谱反射值. 在测量光谱时, 面向太阳, 避免 仪器阴影影响水体的反射光谱. 


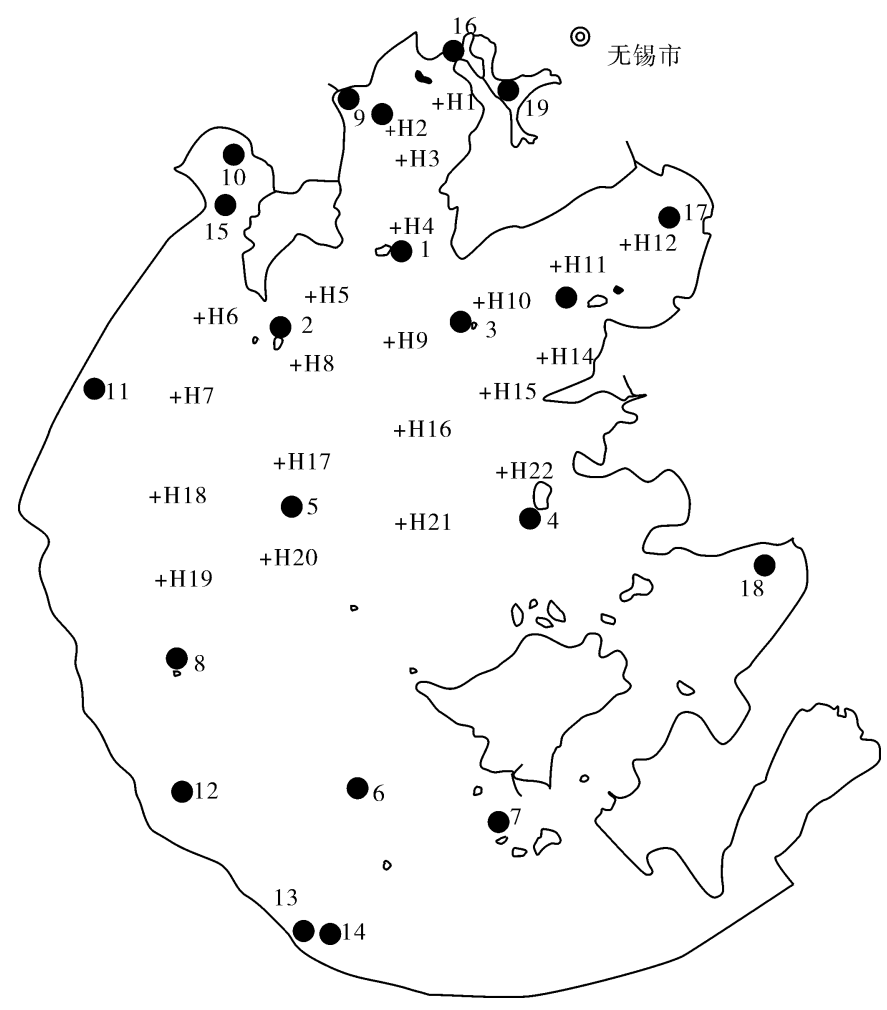

图 1 太湖湖区及水质采样点位置

(•:太湖常规采样点位置; + :2003-11-13 太湖采样点位置)

Fig. 1 Location of samples in Lake Taihu

( : location of routine samples; + : location of samples in 2003-11-13)

考虑到测量中 $400 \mathrm{~nm}$ 以下和 $900 \mathrm{~nm}$ 以上波段中的噪音较大, 实际分析使用的波长范围为 $400-900$ $\mathrm{nm}$ 的反射率. 由于 $\mathrm{H} 8$ 号样点恰好位于蓝藻漂浮区, 其叶绿素浓度值异常高, 因而在此后的分析中没有 使用.

\section{3 遥感影像选择及预处理}

根据太湖的水动力学特征及悬浮物的季节性特征, 选用了四期有代表性的 Landsat TM/ETM 卫星图像, 分别位于春夏秋冬四季,卫星过境时间分别为 2000 年 5 月 4 日 (ETM)、2002 年 8 月 22 日 (TM)、2003 年 11 月 13 日 ( TM) 和 2001 年 1 月 15 日 ( ETM). 首先对一幅 TM 图像 (2003-11-23) 使用 1: 5,0000 地形图进 行几何精纠正 (高斯 - 克里格投影,21 度带), 平均误差为 0.50 个像素. 然后以此校正过的图像对其余的 TM/ETM 图像进行几何精校正,纠正的平均误差和最大误差均小于 1 个像素. TM/ETM 图像受大气的影 响,需要进行辐射校正. $6 \mathrm{~S}$ 模型是目前世界上发展比较完善的大气辐射校正模型之一,非常适合于可见光 - 近红外波段数据,许多研究表明该模型的计算精度比其他模型的高,且计算时间快 ${ }^{[15,20]}$. 同时为了消除 噪音 ${ }^{[6]}$, 进行 $3 \times 3$ 的低通滤波处理, 提取各样点处的反射率值作为遥感数据源

\section{2 结果与分析}

\section{1 悬浮物估测的敏感波段分析}

2003 年 11 月 13 日各采样点处的反射率光谱数据如图 2 所示. 从图中可以看出, 悬浮物散射作用响应 的波长范围比较宽, 以 $500-700 \mathrm{~nm}$ 比较明显; 反射光谱曲线整体上高于清水. 受蓝藻水华的影响, 图中的 反射率光谱带有明显的叶绿素 $\mathrm{a}$ 的光谱特征和悬浮物的光谱特征 ${ }^{[16]}$. 


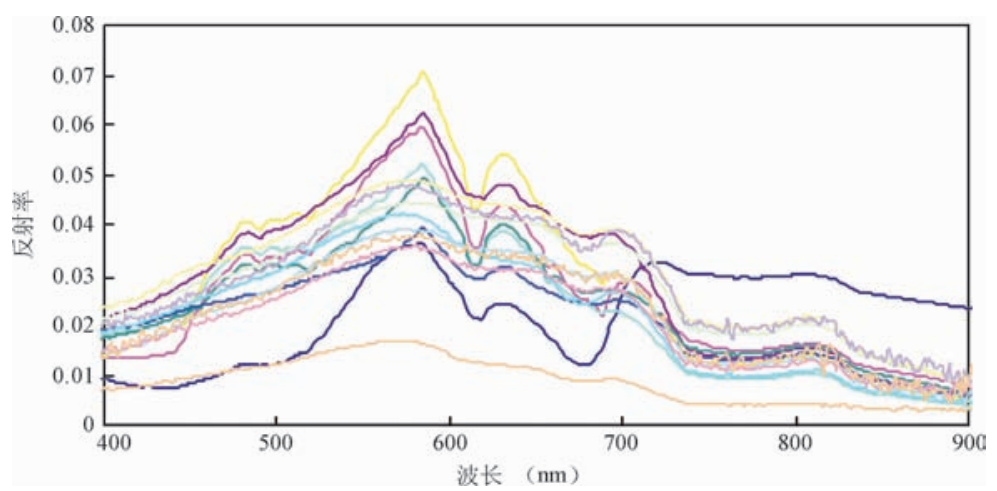

图 2 实测的水面反射率光谱曲线

Fig. 2 Spectral reflectance curves of all sample points, measured on Nov. 13, 2003

光谱中蕴含大量信息,但具体的提取某一段光谱和悬浮物的相关关系,却比较困难,特别是对于内陆水 体而言,不同的地域、不同的时段,通常光谱曲线呈现不同的形态 ${ }^{[21]}$. 由于各单一波段与悬浮物的相关性 都不是很高, 利用相关的波段比值估测悬浮物浓度可以提高估算的精度 ${ }^{[22]}$, 因为反射比可以部分消除周围 环境等背景噪声的干扰,并在一定程度上减小县浮物颗粒物不同特性的影响 ${ }^{[23,24]}$. 杨顶田等 ${ }^{[25]}$ 对太湖梅 梁湾水体中悬浮物光谱分布特征的研究表明与悬浮物含量相关性较好的光谱波长范围在 $400-700 \mathrm{~nm}$, 根 据 Chen ZM 等 ${ }^{[11]}$ 的研究,在 $450 \mathrm{~nm}-700 \mathrm{~nm}$ 波段范围,悬浮物浓度与反射率是一种对数线性关系. 因此, 将 400-900 nm 间的各波段反射率一一作比值运算, 然后分别与悬浮物浓度的对数值 $(\ln S S$ ) 作相关分析, 取组合中各波段对应的最大相关系数为纵轴,相应分子位置的波段值为横轴,结果如图 3.

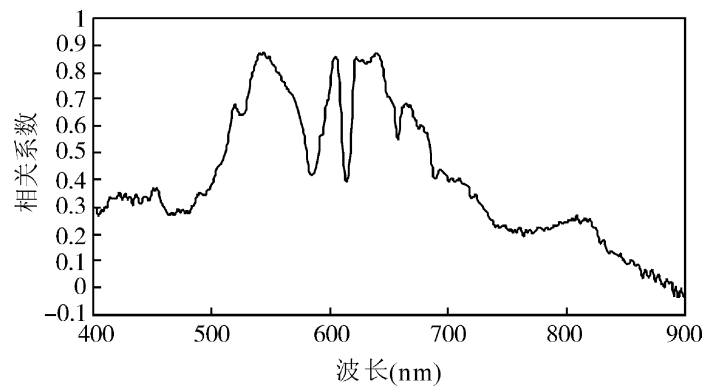

图 $32003-11-13$ 的 $\ln S S$ 与反射率比值的最大相关

Fig. 3 Maximal correlations between $\ln S S$ and spectral reflectance in $2003-11-13$

从图 3 可以看出, $530-570 \mathrm{~nm} 、 620-650 \mathrm{~nm}$ 之 间的相关系数均较高 (相关系数大于 0.7 ), 对应于 Landsat TM/ETM 的 $2 、 3$ 波段. 相关系数的最大值出 现在 R640/R545 nm 处 (相关系数为 0.87 ). 对悬浮 物光谱特性的研究表明 ${ }^{[22,26]}$ : 对悬浮物最为敏感的 波长在 $550 \mathrm{~nm}$ 和 $665 \mathrm{~nm}$, 和我们的研究结果接近. 由于不同季节水质不同,最佳波段比值的具体位置 可能有所偏移,但大体位于 TM/ETM 的第 $2 、 3$ 波段.

\section{2 遥感图像数据与悬浮物浓度的关系}

2.2 .1 遥感数据与悬浮物浓度的相关性分析 2001 $-01-15$ 的 Landsat ETM 图像, 悬浮物含量的实测 数据使用的是 2001 年 1 月 $8-15$ 日的测量数据; 2000-05-04 的 Landsat ETM 图像,悬浮物含量的实测数据使用的是 2000 年 5 月 $8-14$ 日的测量数据, 19 个样点在太湖分布均匀,对全湖的悬浮物分布有一定的代表性. 由于冬、春季水交换强烈, 11 号点 (大浦 口) 、18 号点 (胥口) 位于港口位置 (图 1), 实际选取 17 个采样点进行分析; $2002-08-22$ 的 Landsat TM 图 像, 悬浮物含量的实测数据使用的是 2002 年 9 月 $2-6$ 日的测量数据, 由于 13 号点 (小梅口)、14 号点 (新 港口) 在 TM 图像之外, 实际只有 17 个采样点可以分析;2003-11-13 的 TM 图像, 悬浮物含量的实测数据 使用的是 2003 年 11 月 23 日的测量数据, 剔除叶绿素含量异常高的 H8 号点, 纳人实际计算的样点数为 20 个. 其中,夏、秋季的遥感影像成像日期与采样时间间隔较大,但这期间没有大的风浪,因此可近似认为该 期间悬浮物的分布情况无大的变化. 对样点的悬浮物浓度进行分布检验, 表明对数变换可使得数据更接近 于正态分布. 因此,在模型的构建中, 使用的是悬浮物的对数变换结果,并表示为 $\ln S S$. 为了找出比较合适 的波段或波段组合以估测悬浮物含量, 根据上述敏感波段分析,并参照前人的研究成果 ${ }^{[6,12-18,27]}$ 确定波段 组合形式,计算各单一波段和波段组合与实测的悬浮物浓度对数值的 Pearson 相关系数,结果见表 1 . 
表 1 悬浮物浓度的对数值 $(\ln S S)$ 与波段反射率及波段组合的相关性*

Tab. 1 Correlation between $\ln S S$ and reflectance of TM/ETM bands and band combination

\begin{tabular}{|c|c|c|c|c|c|c|c|c|}
\hline \multirow{2}{*}{ 波段和波段组合相 } & \multicolumn{2}{|c|}{2001 年 1 月 } & \multicolumn{2}{|c|}{2000 年 5 月 } & \multicolumn{2}{|c|}{2002 年 8 月 } & \multicolumn{2}{|c|}{2003 年 11 月 } \\
\hline & 相关系 & 数 & 相关系数 & $p$ & 相关系数 & $p$ & 相关系数 & $p$ \\
\hline$X 1=B 1$ & 0.879 & $1.73 E-06$ & 0.7492 & $2.73 E-04$ & -0.180 & 0.245 & 0.252 & 0.142 \\
\hline$X 2=B 2$ & 0.868 & $3.22 \mathrm{E}-06$ & $0.777 \quad 1$ & 1. $22 \mathrm{E}-04$ & -0.237 & 0.180 & 0.647 & 0.001 \\
\hline$X 3=B 3$ & 0.897 & $5.52 \mathrm{E}-07$ & $0.781 \quad 1$ & $1.07 \mathrm{E}-04$ & -0.088 & 0.368 & 0.712 & 2. $13 E-04$ \\
\hline$X 4=B 4$ & 0.857 & $5.64 \mathrm{E}-06$ & 0.607 & 0.005 & 0.264 & 0.152 & 0.647 & 0.001 \\
\hline$X 5=B 3 / B 2$ & 0.580 & 0.007 & 0.6959 & $9.68 \mathrm{E}-04$ & 0.668 & 0.002 & 0.550 & 0.006 \\
\hline$X 6=B 2 / B 1$ & 0.486 & 0.024 & 0.082 & 0.377 & -0.087 & 0.370 & 0.700 & $2.91 \mathrm{E}-04$ \\
\hline$X 7=B 3 / B 1$ & 0.825 & 2. $27 \mathrm{E}-05$ & 0.602 & 0.005 & 0.252 & 0.165 & 0.788 & $1.85 \mathrm{E}-05$ \\
\hline$X 8=B 4 / B 1$ & 0.564 & 0.009 & -0.222 & 0. 196 & 0.345 & 0.088 & 0.604 & 0.002 \\
\hline$X 9=(B 3+B 4) /(B 1+B 2)$ & 0.628 & 0.003 & 0.184 & 0.240 & 0.470 & 0.029 & 0.684 & 4. $44 \mathrm{E}-04$ \\
\hline$X 10=(B 2+B 3) /(B 2 / B 3)$ & 0.899 & 4. $67 \mathrm{E}-07$ & $0.777 \quad 1$ & 1. $22 \mathrm{E}-04$ & -0.001 & 0.499 & 0.737 & 1. $05 \mathrm{E}-04$ \\
\hline$X 11=(B 3-B 2) /(B 3+B 2)$ & 0.574 & 0.008 & 0.7038 & $8.20 \mathrm{E}-04$ & 0.667 & 0.002 & 0.551 & 0.006 \\
\hline$X 12=(B 3-B 1) /(B 3+B 1)$ & 0. 817 & 3. $15 \mathrm{E}-05$ & 0.621 & 0.004 & 0.287 & 0.132 & 0.803 & $1.01 \mathrm{E}-05$ \\
\hline
\end{tabular}

$*$ 表 1 中, $S S$ 表示悬浮物的浓度值, 单位为 $\mathrm{mg} / \mathrm{L}, B 1-B 5 、 B 7$ 分别表示图像的 $1-5 、 7$ 波段经过大气校 正后的反射率值. $\ln S S$ 是悬浮物浓度的自然对数.

从表 1 中可以看出: 冬季图像的 $1,2,3$ 波段与悬浮物的浓度有较高的相关性 (B3 与 $\ln S S$ 的相关系数 达到 0.897). 夏、秋季的太湖水体受叶绿素和悬浮物的共同影响 ${ }^{[28]}$, 单波段与悬浮物浓度关系较弱, 需要 使用波段组合的方式来减小叶绿素对估测悬浮物的干扰. 对比来看, 不同时期悬浮物与遥感数据的相关关 系不同. 除夏季外, $X 3$ 在其他三个季节与 $\ln S S$ 有着较好的相关性 (相关系数大于 0.712 ), 这与 Zhou W 等 ${ }^{[18]}$ 的研究比较一致,但 $X 3$ 在夏季蓝藻爆发时并不适用,此时与 $\ln S S$ 有着较好相关性的则是 $X 5 、 X 11$; 同 时我们发现 $X 10$ 与 $X 3$ 同样适合于不同水质情况下的悬浮物估测; 此外, $X 7$ 和 Wei YC 等 ${ }^{[17]}$ 提出了悬浮物 归一化指数 X12 比较适合于秋、冬季悬浮物浓度的估测 (相关系数大于 0.788 ). 同一波段或波段组合在不 同数据集中与悬浮物相关性的变化可能是由于悬浮物组成的变化 (无机悬浮物和有机悬浮物的比例变化) 和环境因素 (风力、湖流) 变化引起的.

实测光谱的研究表明,估测悬浮物较好的波段位于 TM/ETM 的第 $2 、 3$ 波段. X10 正是 TM/ETM2、3 波 段的组合,在其表达式中, $B 2+B 3$ 充分考虑了不同浓度悬浮物反射峰的特征, 可有效反映出高、低浓度悬浮 物的光谱特征; $B 2 / B 3$ 是为了抑制叶绿素对低浓度悬浮物遥感信息的干扰作用. 在悬浮物浓度较低、叶绿 素浓度较高的情况下, 因 $B 2$ 处于叶绿素的反射峰, 故 $B 2+B 3$ 将增大, 又因 $B 3$ 处于叶绿素的吸收峰, 故 $B 2 /$ $B 3$ 也将增大, 因此 $(B 2+B 3) /(B 2 / B 3)$ 相应减小, 从而起到抑制叶绿素对悬浮物遥感信息干扰的作用 ${ }^{[27]}$. 因此, 可以认为 $X 10$ 即 $(B 2+B 3) /(B 2 / B 3)$ 是较好的估测悬浮物含量的因子.

2.2 .2 分季节的悬浮物遥感估测模型 根据以上分析结果, 适用于冬季、秋季的悬浮物估测因子有 $X 3$ 、 $X 10 、 X 7 、 X 12$, 适用于春季的悬浮物估算因子有 $X 3 、 X 10$, 适用于夏季悬浮物估算的因子为 $X 5 、 X 11$. 由于 $X 5$ 与 $X 11 、 X 7$ 与 $X 12$ 有着很强的相关性 (相关系数接近于 1 ), 而 $X 11 、 X 12$ 比 $X 5 、 X 7$ 更为稳健 ${ }^{[17]}$, 因而使用 $X 11$ 作为夏季悬浮物浓度的估测因子; 综合考虑各因子与悬浮物相关性的大小, 其他季节的悬浮物估测因 子选用 X10. 提取各期预处理后的 Landsat TM/ETM 图像遥感数据, 与离图像日期最近的实测悬浮物浓度值 分别建立相应的悬浮物遥感估测模型, 然后制作各期太湖湖区的悬浮物浓度分布图.

各期悬浮物遥感估测模型的形式和精度见表 2 ,模型预测值和实测值的对比情况见图 4. 
表 2 模型的形式和精度 ${ }^{1,2)}$

Tab. 2 Models for estimating suspended sediment and their accuracy

\begin{tabular}{|c|c|c|c|c|c|c|c|}
\hline 模型 & 形式 & $N$ & $R^{2}$ & $p$ & $M A E$ & RMSE & $A R E(\%)$ \\
\hline $\begin{array}{c}2001 \text { 年 } 1 \text { 月 } \\
\text { (冬季) }\end{array}$ & $\begin{array}{c}\ln S S=14.656(B 2+B 3) / \\
(B 2 / B 3)+1.661\end{array}$ & 17 & 0.808 & 0.000 & 38.21 & 52.69 & 37.30 \\
\hline $\begin{array}{c}2000 \text { 年 } 5 \text { 月 } \\
\text { (春季) }\end{array}$ & $\begin{array}{c}\ln S S=7.356(B 2+B 3) / \\
(B 2 / B 3)+2.724\end{array}$ & 17 & 0.603 & $2.44 \mathrm{E}-4$ & 25.68 & 34.26 & 33.17 \\
\hline $\begin{array}{c}2002 \text { 年 } 8 \text { 月 } \\
\text { (夏季) }\end{array}$ & $\begin{array}{c}\ln S S=12.253(B 3-B 2) / \\
(B 3+B 2)+3.043\end{array}$ & 17 & 0.444 & 0.003 & 14.46 & 20.62 & 37.14 \\
\hline $\begin{array}{c}2003 \text { 年 } 11 \text { 月 } \\
\text { (秋季) }\end{array}$ & $\begin{array}{c}\ln S S=11.071(B 2+B 3) / \\
(B 2 / B 3)\end{array}$ & 20 & 0.520 & $4.9 E-20$ & 17.80 & 21.66 & 37.53 \\
\hline
\end{tabular}

1)上列各式中, $S S$ 表示悬浮物的浓度值, 单位为 $\mathrm{mg} / \mathrm{L}, \ln S S$ 表示悬浮物取自然对数后的值, $B 2 、 B 3$ 分 别表示 Landsat TM/ETM 图像经过 $6 \mathrm{~S}$ 大气校正、3 $\times 3$ 低通滤波后第 $2 、 3$ 波段的反射率值, $n$ 表示样点个数.

2) $M A E$ :平均绝对误差; $A R E$ :平均相对误差; $R M S E$ :均方根误差.

从表 2 中可以看出,各误差项都较小. 从图 4 中可以看出,各期模型的预测值与实测值都比较接近,夏 季、秋季中个别高浓度的悬浮物估测误差较大. 由于冬季、春季的悬浮物动态范围较大, 故其误差项也较 大. 总体上来看, 上述回归模型可以接受. 以上建立的各季节的悬浮物遥感估测模型中, 冬季的模型是最好 的, 因为冬季能够最大程度的减小叶绿素等因素对于悬浮物估测的干扰 ${ }^{[28]}$. 夏季的模型效果最差,一方面 是由于 8 月是蓝藻爆发的盛季, 叶绿素对悬浮物的估测干扰性较大, 另一方面, 本次使用的夏季图像与水质 实测时间的间隔较长，因而夏季悬浮物估测的效果不好. 2003 年 11 月图像与水质实测时间相差 $10 \mathrm{~d}$ 且水 质采样主要集中于太湖北部, 因而悬浮物估测模型的 $R^{2}$ 也不是很高. 太湖地区春季雨水充沛,湖水的水交 换强烈,对悬浮物的估测也有一定的影响.

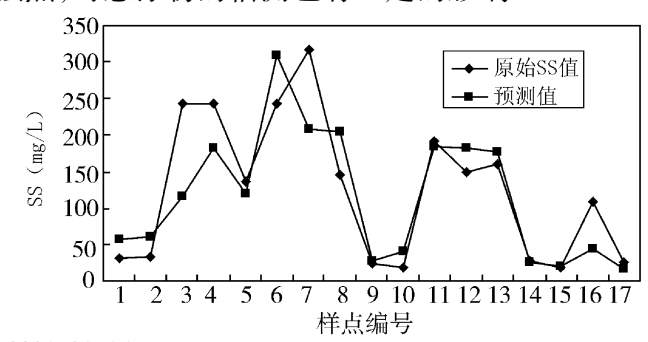

2001-01-15

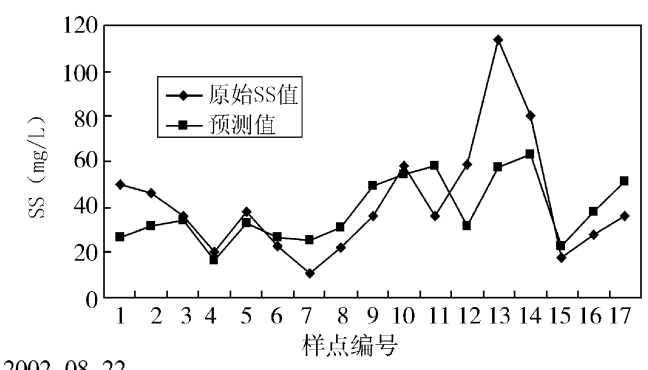

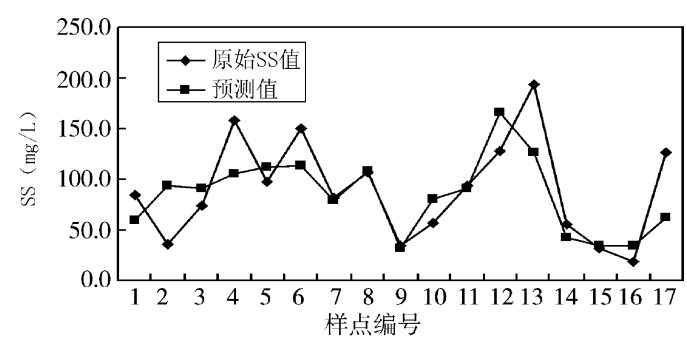

$2000-05-04$

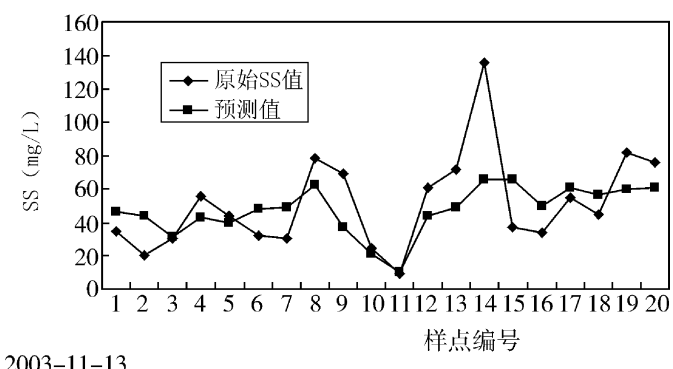

2003-11-13

图 4 各期模型预测值与实测值的对比

Fig. 4 Predicated versus measured suspended sediment concentration in different seasons 


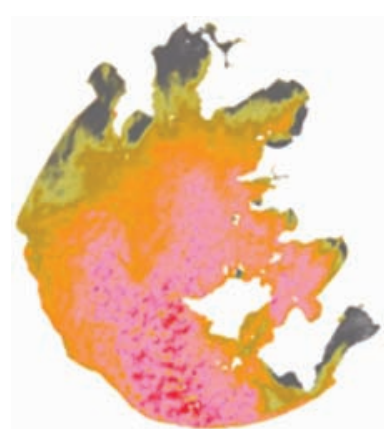

(a) 2001年1月15日 (冬)

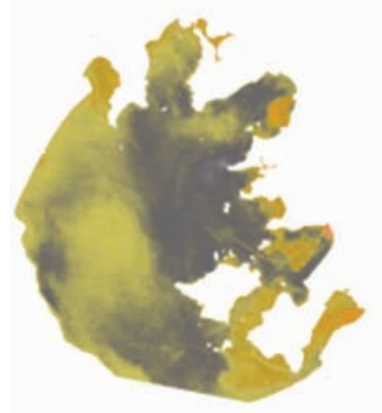

(c) 2002年8月22日 (夏)

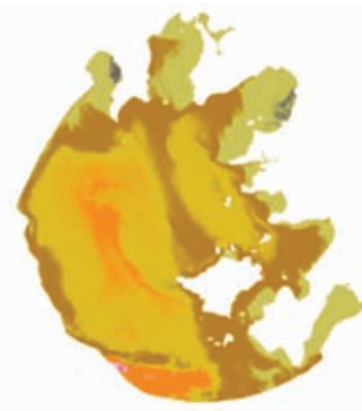

(b) 2000年5月4日 (春)

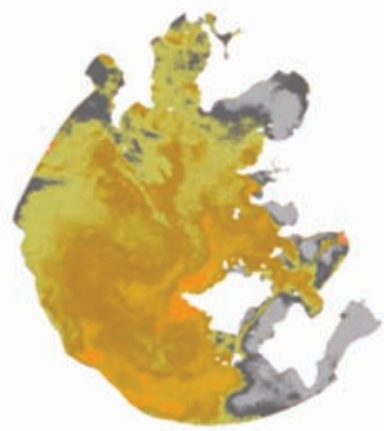

(d) 2003年11月13日 (秋)

图 5 太湖悬浮物浓度分布图

Fig. 5 Distribution of suspended sediment concentrations in Lake Taihu

从图 5 可以看出, 悬浮物的分布有着一定的规律,湖心区和河口区的悬浮物含量都较高, 而悬浮物的最 低值都发生在东太湖. 湖心区是由于其水面开阔, 风速较湖岸大,在风浪作用下底泥发生再悬浮使悬浮物 浓度增高; 河口区由于水交换强烈, 悬浮物浓度因而增高. 西南区苕溪水系坡陡流急, 是太湖主要来水水源 之一, 主流向北由小梅口、大钱口人太湖 ${ }^{[29]}$, 因而西南区的悬浮物含量一直较高. 东太湖悬浮物浓度较低 一方面是由于东太湖位于太湖东南部的一个狭长湖湾, 受风浪的作用小, 另一方面则是由于东太湖的水生 植物对水体起着过滤、净化、消浪、抑制底泥上浮的作用 ${ }^{[19]}$. 季节分布上, 冬、春季悬浮物浓度大于夏、秋 季,这是由于太湖地区夏季、秋季风速小,持续时间短, 风浪作用下的水动力引起的再悬浮过程是控制其季 节变化的主要因素 ${ }^{[19]}$. 这种规律与水质资料分析的结果 ${ }^{[19]}$ 也是一致的, 可见遥感估测的结果基本上反映 了太湖悬浮物时空分布的特征和规律.

\section{3 结语}

本文通过多时态数据 (分布于四个季节), 在前人研究的基础上, 发现了一种新的适合于不同水质情况 下的太湖悬浮物浓度估测的因子 $(B 2+B 3) /(B 2 / B 3)$, 并初步建立了分季节的太湖悬浮物遥感估测模型, 为研究太湖悬浮物的时空变化规律奠定了基础. 研究结果表明:不同的季节条件下,估测悬浮物浓度的最 佳因子是不同的. $(B 3-B 2) /(B 3+B 2)$ 是夏季提取悬浮物浓度的最佳因子, 秋季、冬季提取悬浮物浓度时 可以使用 $B 3 、(B 2+B 3) /(B 2 / B 3)$ 和 $(B 3-B 1) /(B 3+B 1)$, 春季可使用 $B 3$ 和 $(B 2+B 3) /(B 2 / B 3)$ 提取悬 浮物浓度信息. 综合看来, 在除夏季外的其他季节, $(B 2+B 3) /(B 2 / B 3)$ 是最佳的悬浮物估测因子, 对悬浮 物浓度的估测有着较高的精度, 其中冬季的估测效果最佳, 模型为: $\ln S S=14.656 \times(B 2+B 3) /(B 2 / B 3)+$ $1.661\left(R^{2}=0.808\right)$. 式中, $\ln S S$ 表示悬浮物取自然对数后的值, $B 2 、 B 3$ 为 TM/ETM 图像经过 $6 \mathrm{~S}$ 大气校正、 $3 \times 3$ 低通滤波后第 $2 、 3$ 波段的反射率值.

由于本次研究的样本数据还相对较少, 模型的稳定性及代表性还需要进一步验证. 在以后的工作中可 
以加大样点数量, 提高样点的代表性. 随着样本数据的增多, 各季节模型中的参数有可能趋于稳定, 即有望 在没有同步地面数据的情况下通过卫星图像和模型估测当时的悬浮物浓度. 另外, 虽然目前通过实验手段 对单个水质参数如叶绿素、悬浮物、黄色物质等的光谱特性比较了解,但对它们之间的相互影响还不甚了 解, 因而在今后需要加强分析叶绿素及黄色物质对悬浮物浓度估测的影响. 只有分离了各水质参数并明确 了其间的相互影响后,才可能建立更准确更有效的模型.

致谢: 感谢江苏省环境信息中心李旭文和黎刚在本研究中给予的许多帮助, 感谢无锡环境监测中心的专业 技术人员在实地采集数据时提供的帮助,感谢中国科学院南京地理与湖泊研究所的吕恒博士为本次研究提 供了部分数据,感谢中国科学院遥感所薛勇研究员提出的修改意见及国家自然科学基金“基于网格计算平 台的 MODIS 数据气溶胶快速监测建模与中间件研究” (40471091) 项目提供的大数据量计算平台的支持.

\section{4 参考文献}

１１］张运林，秦伯强，陈伟民等. 悬浮物浓度对水下光照和初级生产力的影响. 水科学进展, 2004, 15 (5) :615-620.

[2] 陈晓翔, 丁晓英. 用 FY - 1D 数据估算珠江口海域悬浮泥沙含量. 中山大学学报 (自然科学版), 2004,43(增刊) : $194-196$.

[3] 李四海译. 海洋水色遥感原理与应用——国际海洋水色协调工作组 (IOCCG) 报告. 北京: 海洋出版 社, 2002.

[4] Tassan S. A procedure to determine the particulate content of shallow water from Thematic Mapper data. International Journal of Remote Sensing, 1998, 19(3):557 - 562.

[5] Binding C E, Bowers D G, Mitchelson-Jacob E G. An algorithm for the retrieval of suspended sediment concentrations in the Irish Sea from SeaWiFS ocean colour satellite imagery. International Journal of Remote Sensing, 2003, 24(19):3791 - 3806.

[6] 马荣华, 戴锦芳. 结合 Landsat ETM 与实测光谱估测太湖叶绿素及悬浮物含量. 湖泊科学, 2005,17 (2) :97- 103 .

[7] Binding C E, Bowers D G, Mitchelson-Jacob E G. Estimating suspended sediment concentrations from ocean colour measurements in moderately turbid waters; the impact of variable particle scattering properties. Remote Sensing of Environment, 2005, 94(3) : 373 - 383.

[8] Williams A N, Grabau W E. Sediment concentration mapping in tidal estuaries. Third Earth Resources Technology Satellite-1 Symposium, NASA SP-351, 1973, 1:1347-1386.

[9] Klemas V, Bartlett D, Philpot W et al. Coastal and estuarine studies with ERTS -1 and Skylab. Remote Sensing of Environment, 1974, 3(3):153 - 174.

[10] Munday J C, Alfoldi T T. Landsat test of diffuse reflectance models for aquatic suspended solids measurement. Remote Sensing of Environment, 1979, 8:169-183.

[11] Chen Z M, Hanson J D, Curran P J. The form of the relationship between suspended sediment concentration and spectral reflectance: its implications for the use of Daedalus 1268 data. International Journal of Remote Sensing, 1991, 12(1): $215-222$.

[12] Lathrop R G. Landsat thematic mapper monitoring of turbid inland water quality. Photogrammetric Engineering of Remote Sensing, 1992, 58(4): 465 - 470.

[13 ] Populus J, Hastuti W, Martin J L M et al. Remote sensing as a tool for diagnosis of water quality in Indonesian Seas. Ocean and Coastal Management, 1995, 27(3):197 - 215.

[14] 王学军, 马 延. 应用遥感技术监测和评价太湖水质状况. 环境科学, 2000, 21(6): 65-68.

[15] 吕 恒. 太湖水质多尺度遥感定量监测研究. 中国科学院南京地理与湖泊研究所博士学位论 文, 2004 .

[16] 刘堂友, 匡定波, 尹 球. 湖泊藻类叶绿素 a 和悬浮物浓度的高光谱定量遥感模型研究. 红外与毫米 
波学报, 2004, 23(1): 11-15.

[17] Wei Y C, Huang J Z, Li X W et al. ETM monitoring model on suspended sediment concentration in different seasons in Lake Taihu, China. Proc SPIE, 2005, 6199: 619902.

[18] Zhou W, Wang S, Zhou Y et al. Mapping the concentrations of total suspended matter in Lake Taihu, China, using Landsat-5 TM data. International Journal of Remote Sensing, 2006, 27(6):1177 - 1191.

[19] 张运林, 秦伯强, 陈伟民等. 太湖水体中悬浮物研究. 长江流域资源与环境, 2004, 13 (3): 266 -271 .

[20] Zhao W, Tamura M, Takahashi H. Atmospheric and spectral corrections for estimating surface albedo from satellite data using 6S code. Remote Sensing of Environment, 2000, 76(2) : 202 - 212.

[21] Gin K Y H, Koh S T, Lin II. Spectral irradiance profiles of suspended marine clay for the estimation of suspended sediment concentration in tropical waters. International Journal of Remote Sensing, 2003, 24(16) : $3235-3245$.

[22] Doxaran D, Froidefond J M, Castaing P. A reflectance band ratio used to estimate suspended matter concentrations in sediment-dominated coastal waters. International Journal of Remote Sensing, 2002, 23 ( 23 ) : $5079-5085$.

[23] Doxaran D, Froidefond J M, Lavender S et al. Spectral signature of highly turbid waters: application with SPOT data to quantify suspended particulate matter concentrations. Remote Sensing of Environment, 2002, 81( 1$): 149-161$.

[24] Moore G F, Aiken J, Lavender S J. The atmospheric correction of water colour and the quantitative retrieval of suspended particulate matter in case II waters: application to MERIS. International Journal of Remote Sensing, 1999, 20(9):1713-1733.

[25] 杨顶田，陈伟民，张运林等. 太湖梅梁湾水体中悬浮质及光谱的分布特征. 生态科学, 2002, 21(4)： $289-293$.

[26] 傅克忖, 荒川久幸, 曾宪模. 悬沙水体不同波段反射比的分布特征及悬沙量估算实验研究. 海洋学 报, 1999, 21(3): 134-140.

[27] 李四海, 唐军武, 恽才兴. 河口悬浮泥沙浓度 SeaWiFS 遥感定量模式研究应用. 海洋学报, 2002, 24 (2) $: 51-58$.

[28] 戴昌达, 姜小光, 唐伶俐. 遥感图像应用处理与分析. 北京: 清华大学出版社, 2004.

[29］黄渏平. 太湖水环境及其污染控制. 北京: 科学出版社, 2001. 